$$
Q_{1}=2 x_{1}^{2}-x_{2}^{2}, \quad Q_{2}=x_{1}^{2}-2 x_{2}^{2}
$$

admit the definite linear combination $Q_{1}-Q_{2}=x_{1}^{2}+x_{2}^{2}$, and the corresponding system (5) admits the indefinite solution $B=x_{1} x_{2}$.

EXAMPLE 2. The three forms

$$
Q_{1}=2 x_{1}^{2}-\stackrel{2}{x_{2}}, \quad Q_{2}=x_{1}^{2}-2 x_{2}^{2}, \quad Q_{3}=x_{1} x_{2}
$$

admit the definite linear combination $Q_{1}-Q_{2}-Q_{3}=x_{1}^{2}-x_{1} x_{2}+x_{2}^{2}$, but the corresponding system (5) admits no solution form $B$.

Carnegie Institute of Technology

\title{
NOTE ON A CONJECTURE DUE TO EULER
}

\author{
E. T. BELL
}

Euler's conjecture (1772) that

$$
x_{1}^{n}+\cdots+x_{t}^{n}=x^{n}
$$

where $n$ is an integer greater than 3 and $2<t<n$, has no solution in rational numbers $x_{1}, \cdots, x_{t}, x$ all different from zero, is still unsettled even in its first case, $n=4, t=3$. It may therefore be of some interest to note a solution of this equation for any $n>3$ and any $t>1$ in terms of (irrational) algebraic numbers, which can be made algebraic integers by suitable choice of a homogeneity parameter, all different from zero, all the numbers being polynomials in numbers of degree $2 d$, where $4 d \leqq 2 n-5+(-1)^{n}$. If solutions differing only by a parameter are not considered distinct, there are at least $d^{t-1}$ sets of solutions $x_{1}, \cdots, x_{t}, x$.

The solutions described are

$$
\begin{gathered}
x_{1}=u, \quad x_{2}=r_{t-1} u, \quad x=\left(1+r_{1}\right) \cdots\left(1+r_{t-1}\right) u \\
x_{j}=r_{t-j+1}\left(1+r_{t-j+2}\right)\left(1+r_{t-j+3}\right) \cdots\left(1+r_{t-1}\right) u, \quad j=3, \cdots, t,
\end{gathered}
$$

where $u$ is a parameter and the $r$ 's are any roots, the same or different, of any factor $F_{n}(r)$, irreducible in the field of rational numbers, of

$$
f(r) \equiv \sum_{s=1}^{n-1}(n, s) r^{n-s-1},
$$

Received by the editors July 9, 1942. 
where $(n, s)$ is the binomial coefficient $n ! / s !(n-s)$ !. For, $(r \neq 0)$, $f(r)=0$ implies $(1+r)^{n}=1+r^{n}$; whence the verification is immediate on successive reduction of $x_{1}^{n}+x_{2}^{n}, x_{1}^{n}+x_{2}^{n}+x_{3}^{n}, x_{1}^{n}+\cdots+x_{t}^{n}$. The remarks on $d$ and the number of sets of solutions then follow since $f(r)=0$ is a reciprocal equation, and $F_{n}(r)$ has no multiple roots.

With $y \equiv r+r^{-1}$, the first seven $F_{n}(r)$ are

$$
\begin{array}{ll}
n=4: & 2 y+3 \\
n=5: & y+1 \\
n=6: & 6 y^{2}+15 y+8 \\
n=7: & y+1 \\
n=8: & 4 y^{3}+14 y^{2}+16 y+7 \\
n=9: & 3 y^{3}+9 y^{2}+10 y+5 \\
n=10: & 10 y^{4}+45 y^{3}+80 y^{2}+75 y+32 .
\end{array}
$$

California Institute of Technology 